\title{
Zonotopic fault detection observer for discrete-time descriptor systems considering $\mathcal{H}_{-}$fault sensitivity
}

\author{
Ye Wang ${ }^{a}$, Vicenç Puig ${ }^{b}$, Gabriela Cembrano ${ }^{b, c}$ and Yuxin Zhao ${ }^{a}$ \\ ${ }^{a}$ College of Intelligent Systems Science and Engineering, Harbin Engineering University, Harbin, People's Republic of China; ${ }^{b}$ Advanced Control \\ Systems (SAC) Research Group at Institut de Robòtica i Informàtica Industrial (IRI), CSIC-UPC, Automatic Control Department (ESAII), Universitat \\ Politècnica de Catalunya-BarcelonaTech (UPC), Barcelona, Spain; ' Cetaqua, Water Technology Center, Barcelona, Spain
}

\begin{abstract}
This paper addresses a zonotopic fault detection (FD) observer for discrete-time descriptor systems subject to additive actuator faults. The considered descriptor systems are also perturbed by unknown-but-bounded uncertainties including state disturbances and measurement noise. Under a set-based framework, the effects of uncertainties and faults are separated into two state zonotopes. Based on this decomposition, the FD observer gain is designed to be robust against uncertainties and meanwhile sensitive to faults based on a $\mathcal{H}_{-}$index in a finite-frequency domain. Then, two linear matrix inequality (LMI) conditions are obtained to design an observer that achieves robustness and fault sensitivity at the same time. The generalised KYP lemma is applied to address the fault sensitivity condition. The optimal FD observer gain can be obtained via an offline design procedure. Finally, the proposed fault detection method is applied to a chemical mixing system and the effectiveness is shown.
\end{abstract}

\section{KEYWORDS}

Fault detection; zonotopic observer; robustness; fault sensitivity; discrete-time descriptor systems; linear matrix inequalities; generalised KYP lemma

\section{Introduction}

Fault detection (FD) has played a significant role in the management of critical infrastructure due to the increasing need of maintaining the safety and reliability. Among a large amount of existing methods, model-based FD has been widely investigated, which has also been proved to be a powerful tool applied to a variety of complex systems (see e.g. Blanke et al., 2016; Chen \& Patton, 1999; Ding, 2013; Puig, 2010; Puig et al., 2008; Varga, 2017). The basic idea of implementing a model-based FD method is to generate a residual signal that is used to verify the inconsistency between the system model and measurement information in the presence of occurred faults. These residual generations rely on suitable state observations obtained by an observer. One of the challenges of traditional modelbased FD methods is to define a suitable threshold for FD decision-making. If this threshold is not adequately selected, wrong FD alarms might be triggered.

For the implementation of an FD in real-world applications, robust performance is required in the decision-making stage to allow distinguishing faults from the effects of uncertainties including disturbances and measurement noise. Meanwhile, enhancing the sensitivity to faults is of importance in the FD design. Alternatively, robustness and fault sensitivity can be measured by some performance indices, such as $\mathcal{H}_{\infty}, \mathcal{H}_{2}$ and $\mathcal{H}_{-}$norms (Ding, 2013). In the literature, an increasing number of research works have been carried out for deriving sufficient conditions taking into account the combination of these performance indices by using linear matrix inequalities (LMIs). Some interesting results of $\mathcal{H}_{\infty}, \mathcal{H}_{2}$ and mixed $\mathcal{H}_{-} / \mathcal{H}_{\infty}$ methods for designing a robust FD observer can be found in Ding (2013), Chadli et al. (2013) and Wang et al. (2017).

Set theory has been widely employed in state observations over the last decades, because of the ability to deal with model uncertainties in a guaranteed manner (i.e. producing bounding sets that encloses the worstcase effect of uncertainties) (see Alamo et al., 2005; Combastel, 2015). Considering that system uncertainties are modelled in unknown-but-bounded context, uncertain systems are propagated using a predefined 
geometric set. Different types of sets have been considered in the literature, such as ellipsoids and zonotopes. Some works related to ellipsoidal set-membership approaches can be found in (Chabane et al., 2014; Merhy et al., 2019). Alternatively, zonotopes, known as one of symmetric sets, have simple computational load based on operations involving centre and matrix. Moreover, linear transformation with zonotopes (via the Minkowski sum) can be easily implemented. For a set-based state estimation approach, robustness can be achieved and effects of unknown-but-bounded uncertainties can be explicitly characterised in propagated zonotopes (Alamo et al., 2005). Some existing works have also pointed out its possible application to fault diagnosis framework (see Puig, 2010; Puig et al., 2008; Xu et al., 2014).

From an application point of view, due to mass or energy conservation laws, system behaviours are characterised not only by differential/difference equations for describing system dynamics but also by algebraic equations for representing static relations among some system variables. Such systems are known as descriptor systems, also alternatively named as singular or differential-algebraic systems. The descriptor systems have been used in a variety of applications with critical characteristics, such as drinking water networks (Wang et al., 2017), chemical process (Biegler et al., 2012), aircrafts (Stevens et al., 2016) and electrical systems (Duan, 2010). For these critical complex systems, safety and reliability are always required to be considered in the design of their control systems. Due to this reason, a suitable FD method is a key element for FTC mechanisms that guarantee acceptable performance degradations after possible malfunctions of components inside. Some FD strategies have been studied for different classes of descriptor systems, such as continuous-time linear descriptor systems (Varga, 2017; Yeu et al., 2005), fractionalorder descriptor systems (Komachali \& Shafiee, 2020; Komachali et al., 2019; Nosrati \& Shafiee, 2018).

Taking into account the aforementioned advantages of using the set theory, a set-based FD method is of interest to be developed for descriptor systems. In addition to achieve robustness to uncertainties, the way how to characterise fault sensitivity in a set-based FD method is a relevant issue. In Wang et al. (2017), a preliminary result of combining a set-based FD observer with the $\mathcal{H}_{-}$fault sensitivity has been briefly discussed for discrete-time dynamic systems subject to sensor faults, where the effects of sensor faults are propagated in the centre of the defined residual zonotope. The frequency domain of the faults are not considered in the design of observer gain.

The main contribution of this paper is to propose a zonotopic fault detection (FD) observer for discrete-time descriptor systems subject to additive actuator faults. The considered descriptor systems are also perturbed by unknown-but-bounded uncertainties including state disturbances and measurement noise. Under a set-based framework, the effects of uncertainties and faults are separated into two state zonotopes. Based on this decomposition, the FD observer gain is designed to be robust against uncertainties and meanwhile sensitive to faults based on a $\mathcal{H}_{-}$index. In particular, the frequency of faults (as e.g. oscillatory faults (Goupil, 2010)) is considered in a finite-frequency domain. Two linear matrix inequality (LMI) conditions are obtained to design an observer that achieves robustness and fault sensitivity at the same time. The generalised KYP lemma is applied to address the fault sensitivity condition considering the faults are in a finite-frequency domain. The optimal FD observer gain can be obtained via an offline design procedure. Finally, the proposed fault detection method is applied to a chemical mixing system and the effectiveness is shown.

This paper was motivated by the problem of fault detection (FD) for the class of descriptor systems that can be applied to a large amount of applications, such as drinking/waste water networks, chemical processes, and robot manipulators or arms, etc. Existing approaches for such systems rely on generating a residual signal compared with a predefined threshold to make a decision for the FD alarm. Due to the effects of uncertainties, those approaches could be conservative in the sense that the wrong FD alarm because of the conservativeness of the threshold settings. The proposed approach is based on set theory to overcome the conservativeness from uncertainties, especially by means of zonotopes to characterise the effects of uncertainties. Besides, in this paper, we have also characterised the effects of faults by using zonotopes in order to give a fault sensitivity condition. The proposed approach can be applied in many cyber-physical systems especially including some certain static relations formulated by using algebraic equations.

The remainder of this paper is organised as follows. Some preliminary results are expressed in Section 2. 
The problem statement is formulated in Section 3. The main results including the zonotopic FD observer gain design are presented in Section 4. The application of the proposed method to a chemical mixing system is shown in Section 5. Finally, concluding remarks are drawn in Section 6.

Notation. We use $I_{n}$ to denote an identity matrix of dimension $n$. Note that the dimension $n$ may be dropped when it can be inferred from the context. We use $\mathbf{1}_{m \times n}$ to denote an matrix with all elements of 1 . For a matrix $A, A \succ 0(A \prec 0)$ is said to be positive (negative) definite. We use $\operatorname{tr}(A), \operatorname{rank}(A), A^{\top}$ and $A^{\dagger}$ to denote the trace, rank, (Hermitian) transpose and pseudo-inverse of $A$, respectively. We denote $\mathrm{He}(A)=$ $A+A^{\top}$. We use $\underline{\sigma}(A)$ to denote the minimum singular value of $A$. We also define the following sets $\mathbb{S}^{n}:=$ $\left\{A \in \mathbb{R}^{n \times n} \mid A=A^{\top}\right\}$ and $\mathbb{S}_{\succ 0}^{n}:=\left\{A \in \mathbb{R}^{n \times n} \mid A=\right.$ $\left.A^{\top}, A \succ 0\right\}$. For a vector $z$, the weighted 2-norm of $z$ is denoted by $\|z\|_{W}=\sqrt{z^{\top} W z}$ with a weighting matrix $W \in \mathbb{S}_{\succ 0}^{n}$. The infinity norm of $z$ is denoted by $\|z\|_{\infty}:=\max (z)$. For a signal $z(k), \forall k \in \mathbb{N}$, the $\mathcal{L}_{2}$ norm of $z(k)$ is denoted by $\|z\|_{2}=\sqrt{\sum_{k=0}^{\infty} z(k)^{\top} z(k)}$. Besides, we use $\oplus$ and $\odot$ to denote the Minkowski sum and the linear image. We also use $\star$ to denote a term in a matrix induced by symmetry.

\section{Preliminaries}

In this section, we introduce some necessary definitions, properties and mathematical background that will be used in this paper.

\subsection{Zonotopes}

Definition 2.1 (Zonotope): A zonotope $\mathcal{Z} \subset \mathbb{R}^{n}$ in $n$ dimensional space is defined with its centre $c \in \mathbb{R}^{n}$ and the segment matrix $H \in \mathbb{R}^{n \times r}$ as

$$
\mathcal{Z}=\langle c, H\rangle=\left\{c+H z,\|b\|_{\infty} \leq 1\right\},
$$

where $b \in[-1,+1]^{r}$ is a unit vector of dimension $r$.

Let us denote $\mathbf{B}^{r}=[-1,+1]^{r} \subset \mathbb{R}^{r}$ as a $r$-order hypercube. With the Minkowski sum $\oplus$, the zonotope in (1) can also be defined by $\mathcal{Z}=c \oplus H \mathbf{B}^{r}$. Besides, the following linear properties hold

$$
\begin{aligned}
\left\langle c_{1}, H_{1}\right\rangle \oplus\left\langle c_{2}, H_{2}\right\rangle & =\left\langle c_{1}+c_{2},\left[H_{1}, H_{2}\right]\right\rangle, \\
L \odot\langle c, H\rangle & =\langle L c, L H\rangle,
\end{aligned}
$$

where $L$ is an arbitrary matrix of appropriate dimension.

Definition 2.2 (Interval hull): Given a zonotope $\mathcal{Z}=$ $\langle c, H\rangle \subset \mathbb{R}^{n}$, the interval hull $r s(H) \in \mathbb{R}^{n \times n}$ is defined as an aligned minimum box such that the inclusion property holds: $\langle c, H\rangle \subset\langle c, r s(H)\rangle$, where $r s(H)$ is a diagonal matrix with diagonal elements of $r s(H)_{i, i}=$ $\sum_{j=1}^{r}\left|H_{i, j}\right|, i=1, \ldots, n$.

Definition 2.3 ( $W$-radius): Given a zonotope $\mathcal{Z}=$ $\langle c, H\rangle \subset \mathbb{R}^{n}$ and a weighting matrix $W \in \mathbb{S}_{\succ 0}^{n}$, the $W$-radius of $\mathcal{Z}$ is defined by $\ell_{W}=\max _{\|b\|_{\infty} \leq 1}\|H b\|_{W}^{2}=$ $\max b^{\top} H^{\top} W H b$, where $b$ is the unit vector associ$\|b\|_{\infty} \leq 1$

ated to the zonotope $\mathcal{Z}$.

To reduce the order of a zonotope $\mathcal{Z}=\langle c, H\rangle \subset$ $\mathbb{R}^{n}$, the weighted reduction operator $\downarrow_{q, W}(H)$ proposed in Combastel (2015) is used, where $q$ specifies the maximum number of columns of $H$ and $W \in \mathbb{S}_{\succ 0}^{n}$ is a weighting matrix. The inclusion property $\langle c, H\rangle \subset$ $\left\langle c, \downarrow_{q, W}(H)\right\rangle$ also holds. The procedure for implementing the operator $\downarrow_{q, W}(H)$ is summarised as follows:

- Sort the column of segment matrix $H$ in decreasing order: $\downarrow_{W}(H)=\left[h_{1}, h_{2}, \ldots, h_{r}\right],\left\|h_{j}\right\|_{W}^{2} \geq$ $\left\|h_{j+1}\right\|_{W}^{2}$, where $\left\|h_{j}\right\|_{W}$ is the weighted 2-norm of $h_{j}$.

- Take the first $q$-column of $\downarrow_{W}(H)$ and enclose a set $H_{<}$generated by remaining columns into a smallest box (interval hull) as follows:

$$
\begin{aligned}
& \text { If } r \leq q \text {, then } \downarrow_{q, W}(H)=\downarrow_{W}(H), \\
& \text { Else } \downarrow_{q, W}(H)=\left[H_{>}, r s\left(H_{<}\right)\right] \in \mathbb{R}^{n \times(q+n)}, \\
& \quad H_{>}=\left[h_{1}, \ldots, h_{q}\right], \quad H_{<}=\left[h_{q+1}, \ldots, h_{r}\right] .
\end{aligned}
$$

\section{2. $\mathcal{H}_{-}$index and generalised $K Y P$ lemma}

We now introduce the definition of the $\mathcal{H}_{-}$index and generalised KYP lemma in the following.

Definition $2.4\left(\mathcal{H}_{-}\right.$index of discrete-time systems (Ding, 2013)): Given a transfer function $G_{y u}(z)$ of discrete-time systems as $G_{y u}(z)=\mathcal{C}(z I-\mathcal{A})^{-1} \mathcal{B}+$ $\mathcal{D}$ between signals $y(k)$ and $u(k), \forall k \in \mathbb{N}$ with $z=e^{j \theta}$, 
Table 1. The selections of matrices $\Xi$ in different frequency domains.

\begin{tabular}{|c|c|c|c|}
\hline & LF & MF & $\mathrm{HF}$ \\
\hline$\Theta$ & $|\theta| \leq \theta_{l}$ & $\theta_{1} \leq \theta \leq \theta_{2}$ & $|\theta| \geq \theta_{h}$ \\
\hline$\Xi$ & {$\left[\begin{array}{cc}-P & Q \\
Q & P-2 \cos \left(\theta_{l}\right) Q\end{array}\right]$} & {$\left[\begin{array}{cc}-P & e^{\bar{j} \theta} c Q \\
e^{-j \theta} c Q & P-2 \cos \left(\theta_{W}\right) Q\end{array}\right]$} & {$\left[\begin{array}{ll}-P & -Q \\
-Q P+2 \cos \left(\theta_{h}\right) Q\end{array}\right.$} \\
\hline
\end{tabular}

LF: low-frequency domain, MF: middle-frequency domain, HF: high-frequency domain. $\theta_{1}, \theta_{1}, \theta_{2}$ and $\theta_{h}$ are low-, pass-band, and high- cut-off frequencies selected according to the fault frequency contents.

the $\mathcal{H}_{-}$index of $G_{y u}(z)$ is defined by

$$
\left\|G_{y u}(z)\right\|_{-}:=\inf _{u \neq 0} \frac{\|y\|_{2}}{\|u\|_{2}}=\inf _{\theta} \underline{\sigma}\left(G_{y u}\left(e^{j \theta}\right)\right) .
$$

By this definition, the $\mathcal{H}_{-}$index between signals $y(k)$ and $u(k), k \in \mathbb{N}$ can also be presented by $\left\|G_{y u}(z)\right\|_{-} \geq \beta$ with $\beta>0$, that is

$$
\sum_{k=0}^{\infty} y(k)^{\top} y(k) \geq \beta^{2} \sum_{k=0}^{\infty} u(k)^{\top} u(k) .
$$

Lemma 2.5 (Generalized KYP lemma for discretetime systems from Iwasaki and Hara (2005)): Given a transfer function $G(z)$ of discrete-time systems as $G(z)=\mathcal{C}(z I-\mathcal{A})^{-1} \mathcal{B}+\mathcal{D}$ with $z=e^{j \theta}$, a symmetric matrix $\Pi$ of appropriate dimension. The following statements are equivalent

(1) For a finite-frequency domain $\forall \theta \in \Theta$, the following condition holds

$$
\left[\begin{array}{c}
G\left(e^{j \theta}\right) \\
I
\end{array}\right]^{\top} \Pi\left[\begin{array}{c}
G\left(e^{j \theta}\right) \\
I
\end{array}\right] \prec 0, \forall \theta \in \Theta .
$$

(2) There exist Hermitian matrices $P$ and $Q$ such that $Q \succ 0$ and

$$
\begin{aligned}
& {\left[\begin{array}{cc}
\mathcal{A} & \mathcal{B} \\
I & 0
\end{array}\right]^{\top} \Xi\left[\begin{array}{cc}
\mathcal{A} & \mathcal{B} \\
I & 0
\end{array}\right]+\left[\begin{array}{cc}
\mathcal{C} & \mathcal{D} \\
0 & I
\end{array}\right]^{\top}} \\
& \Pi\left[\begin{array}{cc}
\mathcal{C} & \mathcal{D} \\
0 & I
\end{array}\right] \prec 0,
\end{aligned}
$$

where the selections of $\Xi$ are presented in Table 1, and $\theta_{c}=\frac{\theta_{1}+\theta_{2}}{2}, \theta_{w}=\frac{\theta_{2}-\theta_{1}}{2}$.

\section{Problem statement}

Consider the class of discrete-time descriptor system subject to additive actuator faults as

$$
E x(k+1)=A x(k)+B u(k)+B_{w} w(k)+F f(k),
$$

$$
y(k)=C x(k)+D_{v} v(k)
$$

where $x \in \mathbb{R}^{n}, u \in \mathbb{R}^{m}$ and $y \in \mathbb{R}^{p}$ denote the state, the input and the output vectors, $w \in \mathbb{R}^{m_{w}}, v \in \mathbb{R}^{m_{v}}$ and $f \in \mathbb{R}^{m_{f}}$ denote the state disturbance, the measurement noise and the additive fault vectors. $k \in \mathbb{N}$ denotes a time instant. Besides, $A \in \mathbb{R}^{n \times n}, B \in \mathbb{R}^{n \times m}$, $B_{w} \in \mathbb{R}^{n \times m_{w}}, F \in \mathbb{R}^{n \times m_{f}}, C \in \mathbb{R}^{p \times n}$ and $D_{v} \in \mathbb{R}^{p \times m_{v}}$ are the system matrices. From the descriptor context, the matrix $E \in \mathbb{R}^{n \times n}$ may be singular, that is, $\operatorname{rank}(E) \leq n$.

The following assumptions are made, which will be used in this paper.

Assumption 3.1: The disturbance vector $w(k)$ and the noise vector $v(k)$ are unknown but bounded by the zonotopes

$$
w(k) \in\left\langle 0, I_{m_{w}}\right\rangle, \quad v(k) \in\left\langle 0, I_{m_{v}}\right\rangle, \quad \forall k \in \mathbb{N} .
$$

Assumption 3.2: The initial state $x(0)$ is bounded by the initial zonotope $x_{0} \in\left\langle c_{0}, H_{0}\right\rangle \subset \mathbb{R}^{n}$, which can be obtained as e.g. from the physical limits of the states or some other physical a priori knowledge about the system.

To design an observer for the descriptor system (7), the following assumption is employed.

Assumption 3.3: Matrices E, C satisfy the following rank condition:

$$
\operatorname{rank}\left[\begin{array}{l}
E \\
C
\end{array}\right]=n
$$

Remark 3.1: The condition (9) has been widely used for observer design for descriptor systems in some existing works (see Wang et al., 2018, 2012).

Since the rank condition (9) holds from Assumption 3.3, there exist two non-empty matrices $T \in \mathbb{R}^{n \times n}$ and $N \in \mathbb{R}^{n \times p}$ such that

$$
T E+N C=I_{n} .
$$

The general solution of $T$ and $N$ satisfying (10) is given by

$$
\begin{gathered}
T=\Psi^{\dagger} \alpha_{1}+\bar{S}\left(I_{n+p}-\Psi \Psi^{\dagger}\right) \alpha_{1}, \\
N=\Psi^{\dagger} \alpha_{2}+\bar{S}\left(I_{n+p}-\Psi \Psi^{\dagger}\right) \alpha_{2},
\end{gathered}
$$


where

$$
\Psi=\left[\begin{array}{c}
E \\
C
\end{array}\right], \quad \alpha_{1}=\left[\begin{array}{c}
I_{n} \\
0
\end{array}\right], \quad \alpha_{2}=\left[\begin{array}{c}
0 \\
I_{p}
\end{array}\right],
$$

and $\bar{S}$ is an arbitrary matrix of appropriate dimension.

We first recall the state bounding zonotope for the descriptor system (7) with $f \equiv 0$ from (Wang et al., 2018, Theorem 5) as the following proposition.

Proposition 3.4: Consider the descriptor system (7) with $f \equiv 0, w(k) \in\left\langle 0, I_{m_{w}}\right\rangle$ and $v(k) \in\left\langle 0, I_{m_{v}}\right\rangle, \forall k \in$ $\mathbb{N}$. Suppose that $x(k) \in\langle\hat{c}(k), \hat{H}(k)\rangle \subseteq\langle\hat{c}(k), \bar{H}(k)\rangle$ with $\bar{H}=\downarrow_{q, W}(\hat{H})$ at time step $k \in \mathbb{N}$. Then, the state bounding zonotope can be recursively defined by $x(k+$ $1) \in\langle\hat{c}(k+1), \hat{H}(k+1)\rangle$, where

$$
\begin{aligned}
\hat{c}(k+1)= & (T A-G C) \hat{c}(k)+T B u(k) \\
& +G y(k)+N y(k+1), \\
\hat{H}(k+1)= & {\left[(T A-G C) \bar{H}(k), T B_{w},-G D_{v},-N D_{v}\right], }
\end{aligned}
$$

where $G \in \mathbb{R}^{n \times p}$ is a time-varying observer gain.

Proof: The proof can be found in (Wang et al., 2018, Theorem 5) and hence omitted here.

From the state zonotope $\hat{x}(k) \in\langle\hat{c}(k), \hat{H}(k)\rangle, \forall k \in$ $\mathbb{N}$, we define the zonotopic FD observer for descriptor system (7) as the residual zonotope $\left\langle c_{r}(k), H_{r}(k)\right\rangle \subset$ $\mathbb{R}^{p}$ with

$$
\begin{aligned}
c_{r}(k) & =y(k)-C \hat{c}(k), \\
H_{r}(k) & =\left[C \hat{H}(k), D_{v}\right] .
\end{aligned}
$$

In this paper, the goal of designing an FD observer gain $G$ is to make the zonotopic FD observer (13) robust against uncertainties and meanwhile sensitive to faults. Since the zonotopic FD observer is implemented by the residual zonotope as in (13), it is indeed a linear transformation of the state bounding zonotope $\hat{x}(k) \in\langle\hat{c}(k), \hat{H}(k)\rangle, \forall k \in \mathbb{N}$. Hence, in the following section, we will discuss about the decomposition of state bounding zonotope to separate the effects of unknown-but-bounded uncertainties and occurred faults. Based on this decomposition, the design conditions of the FD observer gain $G$ will be addressed.

\section{Main results}

We now present the main results of zonotopic FD observer for the descriptor system (7) and the design of the FD observer gain.

\subsection{Zonotopic observer decomposition}

With matrices $T$ and $N$ satisfying (10), from (7b), we have

$$
N C x(k+1)=N y(k+1)-N D_{v} v(k+1) .
$$

Pre-multiplying (7a) by $T$ and adding (14), we obtain the reformulated descriptor dynamics

$$
\begin{aligned}
x(k+1)= & T A x(k)+T B u(k)+T B_{w} w(k) \\
& +T F f(k)+N y(k+1)-N D_{v} v(k+1) .
\end{aligned}
$$

For $\hat{x}(k) \in\langle\hat{c}(k), \hat{H}(k)\rangle$ with $\forall k \in \mathbb{N}$, let us define the state estimation error as $e(k)=x(k)-\hat{c}(k)$. Then, with (15), the state estimation error dynamics can be formulated as

$$
\begin{aligned}
e(k+1)= & (T A-G C) e(k)+T B_{w} w(k)+T F f(k) \\
& -G D_{v} v(k)-N D_{v} v(k+1) .
\end{aligned}
$$

From (16), it can be seen that the effect of occurred fault $f(k)$ exists. Suppose that $x(k) \in\langle c(k), H(k)\rangle=$ $\left\{\langle\hat{c}(k), \hat{H}(k)\rangle \oplus\left\langle c_{f}(k), 0\right\rangle\right\}$ at time step $k$, where $c_{f}(k) \in$ $\mathbb{R}^{n}$ is the centre of the zonotope only affected by faults and we assume that $c_{f}(0)=0$ at time step $k=0$. We now define the decomposition of the state bounding zonotope in two zonotopes that consider the separate effects of uncertainties and faults in the following theorem.

Theorem 4.1 (State bounding zonotope decomposition): Consider the descriptor system (7) with $f \not \equiv 0$, $w(k) \in\left\langle 0, I_{m_{w}}\right\rangle$ and $v(k) \in\left\langle 0, I_{m_{v}}\right\rangle, \forall k \in \mathbb{N}$. Suppose that $x(k) \in\langle c(k), H(k)\rangle=\left\{\langle\hat{c}(k), \hat{H}(k)\rangle \oplus\left\langle c_{f}(k), 0\right\rangle\right\}$ at time step $k \in \mathbb{N}$. Then, the uncertain state $x(k+1)$ at time step $k+1$ is bounded by the zonotope in the decomposition form

$$
\begin{aligned}
x(k+1) & \in\langle c(k+1), H(k+1)\rangle \\
& =\left\{\langle\hat{c}(k+1), \hat{H}(k+1)\rangle \oplus\left\langle c_{f}(k+1), 0\right\rangle\right\},
\end{aligned}
$$


where $\hat{c}(k+1)$ and $\hat{H}(k+1)$ as in (12) and

$$
c_{f}(k+1)=(T A-G C) c_{f}(k)+T F f(k) .
$$

Proof: From $\quad x(k) \in\langle c(k), H(k)\rangle=\{\langle\hat{c}(k), \hat{H}(k)\rangle$ $\left.\oplus\left\langle c_{f}(k), 0\right\rangle\right\}$ at time step $k$, we know $c(k)=\hat{c}(k)+$ $c_{f}(k)$ and $e(k)=x(k)-\hat{c}(k) \in\left\langle c_{f}(k), \hat{H}(k)\right\rangle$. Then, we have that $x(k+1)=\hat{c}(k+1)+e(k+1)$ at time step $k+1$. Considering $w(k) \in\left\langle 0, I_{m_{w}}\right\rangle, v(k) \in\left\langle 0, I_{m_{v}}\right\rangle$, $\forall k \in \mathbb{N}$ with $e(k+1)$ in (16), we can derive that

$$
\begin{aligned}
& x(k+1) \\
& \in\langle c(k+1), H(k+1)\rangle \\
&=\left\{\langle\hat{c}(k+1), \hat{H}(k+1)\rangle \oplus\left\langle c_{f}(k+1), 0\right\rangle\right\} \\
&=\langle\hat{c}(k+1), 0\rangle \oplus\left((T A-G C) \odot\left\langle c_{f}(k), \hat{H}(k)\right\rangle\right) \\
& \oplus\left(T B_{w} \odot\left\langle 0, I_{m_{w}}\right\rangle\right) \oplus\langle T F f(k), 0\rangle \\
& \oplus\left(-G D_{v} \odot\left\langle 0, I_{m_{v}}\right\rangle\right) \oplus\left(-N D_{v} \odot\left\langle 0, I_{m_{v}}\right\rangle\right) .
\end{aligned}
$$

By applying the properties in (2), we obtain $\hat{c}(k+1)$ and $\hat{H}(k+1)$ as in (12) and $c_{f}(k+1)$ as in (18).

Based on the decomposition in Theorem 4.1, it can be seen that the effects of uncertainties and faults are separated. Specifically, the zonotopes $\langle\hat{c}(k), \hat{H}(k)\rangle$, $\forall k \in \mathbb{N}$ are only affected by state disturbance $w(k)$ and measurement noise $v(k)$ while the zonotopes $\left\langle c_{f}(k), 0\right\rangle$, $\forall k \in \mathbb{N}$ are only affected by additive actuator faults $f(k)$.

From Theorem 4.1, we can also obtain the decomposition of zonotopic FD observer (13). Taking into account $\quad x(k) \in\langle c(k), H(k)\rangle=\left\{\langle\hat{c}(k), \hat{H}(k)\rangle \oplus\left\langle c_{f}\right.\right.$ $(k), 0\rangle\}, v(k) \in\left\langle 0, I_{m_{v}}\right\rangle$ and $(7 \mathrm{~b})$, by setting $r(k)=$ $y(k)-C \hat{c}(k)$, we can derive

$$
\begin{aligned}
r(k) \in & \left\langle c_{r}(k), H_{r}(k)\right\rangle \\
= & (C \odot\langle\hat{c}(k), \hat{H}(k)\rangle) \oplus\left(C \odot\left\langle c_{f}(k), 0\right\rangle\right) \\
& \oplus\left(D_{v} \odot\left\langle 0, I_{m_{v}}\right\rangle\right) \oplus(-C \odot\langle\hat{c}(k), 0\rangle) .
\end{aligned}
$$

Then, by applying the properties in (2), we obtain the decomposition form of the zonotopic FD observer $r(k) \in\left\langle c_{r}(k), H_{r}(k)\right\rangle$, where

$$
\begin{aligned}
c_{r}(k) & =C c_{f}(k), \\
H_{r}(k) & =\left[C \hat{H}(k), D_{v}\right] .
\end{aligned}
$$

Based on the decomposition in (19), the effect of faults is characterised by the centre $c_{r}(k)$ while uncertainties are propagated into the zonotope segment matrix $H_{r}(k), \forall k \in \mathbb{N}$. Besides, as defined in (19b), $H_{r}(k)$ can also be regarded as a linear transformation of $\hat{H}(k)$.

Remark 4.1: The structure of the zonotopic FD observer for the descriptor system (7) is defined in (13) that will be used for the FD implementation. The decomposition form (19) is used for the analysis and the design for the FD observer gain $G$.

\subsection{Zonotopic fault detection observer gain design}

We now present the LMI conditions that allow achieving robustness against uncertainties and sensitivity to faults for descriptor system (7). Based on the state bounding zonotope decomposition, the effects of uncertainties and faults are separated in two zonotopes that will be used for addressing robustness and fault sensitivity.

From the analysis in (19), we formulate the robustness condition by minimising the size of the zonotope $\langle\hat{c}(k), \hat{H}(k)\rangle$. We use the $W$-radius to measure the size of this zonotope. On the other hand, we derive the fault sensitivity condition in terms of the centre $c_{f}(k)$ using the $\mathcal{H}_{-}$index.

\subsubsection{Robustness condition}

From Definition 2.3, with a weighing matrix $W \in \mathbb{S}_{\succ 0}^{n}$, the $W$-radius of the zonotope $\langle\hat{c}(k), \hat{H}(k)\rangle$ can be formulated as

$$
\ell_{W}(k)=\max _{\|\bar{z}\|_{\infty} \leq 1}\|\hat{H}(k) \bar{z}\|_{W}^{2} .
$$

Considering uncertainties (disturbances and noise) are propagated and bounded in the zonotope $\langle\hat{c}(k)$, $\hat{H}(k)\rangle, \forall k \in \mathbb{N}$, minimising the size of this zonotope reduces the effect of uncertainties. Following the setbased framework from Wang et al. (2018), a zonotope minimisation criterion is introduced in the following.

Proposition 4.2 ( $W$-radius minimization criterion): Consider the descriptor system (7), the zonotope $\langle\hat{c}(k), \hat{H}(k)\rangle, v \forall k \in \mathbb{N}$ and its $W$-radius defined in (20), two scalars $\gamma \in(0,1)$ and $\epsilon>0$. If there exists a matrix $W \in \mathbb{S}_{\succ 0}^{n}$ such that a minimisation criterion is defined as

$$
\ell_{W}(k+1) \leq \gamma \ell_{W}(k)+\epsilon, \quad \forall k \in \mathbb{N},
$$

then the $W$-radius is ultimately bounded by $\ell_{W}(\infty) \leq$ $\frac{\epsilon}{1-\gamma}$ when $k \rightarrow \infty$. 
Proof: With $\ell_{W}$ in (20), the minimisation criterion in (21) holds $\forall k \in \mathbb{N}$. When $k \rightarrow \infty$, we have $\ell_{W}(\infty) \leq \gamma \ell_{W}(\infty)+\epsilon$, which implies the ultimate boundedness $\ell_{W}(\infty) \leq \frac{\epsilon}{1-\gamma}$.

Remark 4.2: Note that here a particular criterion ( $W$-radius, also called $P$-radius, as introduced in Le et al., 2013, Wang et al., 2018), widely used in the zonotope literature has been used for bounding the effect of the uncertainty. There exist other possible criterion that could be considered. The analysis of the effect selection of in the fault detection performance will be explored in future research.

Based on (21), we now formulate the LMI condition to address robustness of the zonotopic FD observer in the following theorem.

Theorem 4.3 (Robustness condition): Given the descriptor system (7), matrices $T \in \mathbb{R}^{n \times n}$ and $N \in$ $\mathbb{R}^{n \times p}$ satisfying (10). If there exist matrices $W \in$ $\mathbb{S}_{\succ 0}^{n}, Y \in \mathbb{R}^{n \times p}$, diagonal matrices $\Gamma \in \mathbb{S}_{\succ 0}^{m_{w}}, \Upsilon \in \mathbb{S}_{\succ 0}^{m_{v}}$ and $\Omega \in \mathbb{S}_{\succ 0}^{m_{v}}$, and two scalars $\gamma \in(0,1)$ and $\epsilon>0$ such that

$$
\operatorname{tr}(\Gamma)+\operatorname{tr}(\Upsilon)+\operatorname{tr}(\Omega)<\epsilon,
$$

$$
\left[\begin{array}{ccccc}
\gamma W & \star & \star & \star & \star \\
W T A-Y C & W & \star & \star & \star \\
0 & D_{w}^{\top} T^{\top} W^{\top} & \Gamma & \star & \star \\
0 & D_{v}^{\top} Y^{\top} & 0 & \Upsilon & \star \\
0 & D_{v}^{\top} N^{\top} W^{\top} & 0 & 0 & \Omega
\end{array}\right] \succ 0,
$$

then (12) is robustly stable and the W-radius minimisation criterion in (21) holds.

Proof: From $\ell_{W}(k)$ in (20), (21) can be reformulated as

$$
\max _{\|z\|_{\infty} \leq 1}\|\hat{H}(k+1) z\|_{W}^{2} \leq \max _{\|\bar{z}\|_{\infty} \leq 1} \gamma\|\hat{H}(k) \bar{z}\|_{W}^{2}+\epsilon .
$$

Let us set $z=\left[\bar{z}^{\top}, b_{1}^{\top}, b_{2}^{\top}, b_{3}^{\top}\right]^{\top}$ with $b_{1} \in \mathbf{B}^{m_{w}}, b_{2} \in$ $\mathbf{B}^{m_{v}}$ and $b_{3} \in \mathbf{B}^{m_{v}}$. For any $z$ such that $\|z\|_{\infty} \leq 1$, we obtain a sufficient condition from the above inequality

$$
\|\hat{H}(k+1) z\|_{W}^{2}-\gamma\|\hat{H}(k) \bar{z}\|_{W}^{2}-\epsilon<0 .
$$

Recalling $\hat{H}(k+1)$ in $(12 \mathrm{~b})$ and setting $Y=W G$, we denote

$$
R=\left[W T A-Y C, W T D_{w}, Y D_{v}, W N D_{v}\right] .
$$

Then, (23) can be rewritten as

$$
\begin{aligned}
& {\left[\begin{array}{c}
\hat{H}(k) \bar{z} \\
b_{1} \\
b_{2} \\
b_{3}
\end{array}\right]^{\top} R^{\top} W^{-1} R\left[\begin{array}{c}
\hat{H}(k) \bar{z} \\
b_{1} \\
b_{2} \\
b_{3}
\end{array}\right]} \\
& -\bar{z}^{\top} \hat{H}(k)^{\top} \gamma W \hat{H}(k) \bar{z}-\epsilon<0 .
\end{aligned}
$$

If $\Gamma \in \mathbb{S}_{\succ 0}^{m_{w}}, \Upsilon \in \mathbb{S}_{\succ 0}^{m_{v}}$ and $\Omega \in \mathbb{S}_{\succ 0}^{m_{v}}$ are diagonal and positive-definite matrices, then the following conditions hold

$$
\begin{aligned}
& \operatorname{tr}(\Gamma) \geq b_{1}^{\top} \Gamma b_{1}, \forall b_{1} \in \mathbf{B}^{m_{w}}, \\
& \operatorname{tr}(\Upsilon) \geq b_{2}^{\top} \Upsilon b_{2}, \forall b_{2} \in \mathbf{B}^{m_{v}}, \\
& \operatorname{tr}(\Omega) \geq b_{3}^{\top} \Omega b_{3}, \forall b_{3} \in \mathbf{B}^{m_{v}} .
\end{aligned}
$$

Together with (25) and (26), we can obtain a sufficient condition

$$
\begin{aligned}
& {\left[\begin{array}{c}
\hat{H}(k) \bar{z} \\
b_{1} \\
b_{2} \\
b_{3}
\end{array}\right]^{\top} R^{\top} W^{-1} R\left[\begin{array}{c}
\hat{H}(k) \bar{z} \\
b_{1} \\
b_{2} \\
b_{3}
\end{array}\right]-\bar{z}^{\top} \hat{H}(k)^{\top} \gamma W \hat{H}(k) \bar{z}} \\
& \quad+\operatorname{tr}(\Gamma)-b_{1}^{\top} \Gamma b_{1}+\operatorname{tr}(\Upsilon)-b_{2}^{\top} \Upsilon b_{2} \\
& \quad+\operatorname{tr}(\Omega)-b_{3}^{\top} \Omega b_{3}-\epsilon<0,
\end{aligned}
$$

for any $\bar{z}, b_{1}, b_{2}$ and $b_{3}$ such that $\|\bar{z}\|_{\infty} \leq 1,\left\|b_{1}\right\|_{\infty} \leq$ $1,\left\|b_{2}\right\|_{\infty} \leq 1$ and $\left\|b_{3}\right\|_{\infty} \leq 1$.

If (22a) holds, then we have

$$
\begin{aligned}
& {\left[\begin{array}{c}
\hat{H}(k) \bar{z} \\
b_{1} \\
b_{2} \\
b_{3}
\end{array}\right]^{\top}\left(\left[\begin{array}{cccc}
\gamma W & 0 & 0 & 0 \\
0 & \Gamma & 0 & 0 \\
0 & 0 & \Upsilon & 0 \\
0 & 0 & 0 & \Omega
\end{array}\right]-R^{\top} W^{-1} R\right)} \\
& {\left[\begin{array}{c}
\hat{H}(k) \bar{z} \\
b_{1} \\
b_{2} \\
b_{3}
\end{array}\right]>0,}
\end{aligned}
$$

for any $\bar{z}, b_{1}, b_{2}$ and $b_{3}$ such that $\|\bar{z}\|_{\infty} \leq 1,\left\|b_{1}\right\|_{\infty} \leq$ $1,\left\|b_{2}\right\|_{\infty} \leq 1$ and $\left\|b_{3}\right\|_{\infty} \leq 1$. Then, by applying the Schur complement, we obtain

$$
\left[\begin{array}{ccccc}
\gamma W & \star & \star & \star & \star \\
0 & \Gamma & \star & \star & \star \\
0 & 0 & \Upsilon & \star & \star \\
0 & 0 & 0 & \Omega & \star \\
W T A-Y C & W T D_{w} & Y D_{v} & W N D_{v} & W
\end{array}\right] \succ 0 .
$$


Define a linear transformation matrix

$$
M=\left[\begin{array}{lllll}
I & 0 & 0 & 0 & 0 \\
0 & 0 & I & 0 & 0 \\
0 & 0 & 0 & I & 0 \\
0 & 0 & 0 & 0 & I \\
0 & I & 0 & 0 & 0
\end{array}\right] .
$$

Finally, pre-multiplying (27) by $M^{\top}$ and postmultiplying it by $M$, we thus obtain (22b).

Remark 4.3: The condition (22b) implies

$$
\left[\begin{array}{cc}
\gamma W & \star \\
W T A-Y C & W
\end{array}\right] \succ 0,
$$

which is related to the standard design result of a stabilizing observer gain for descriptor systems (see Wang et al., 2018, Proposition 2; Wang et al., 2012, Theorem 1).

\subsection{2. $\mathcal{H}_{-}$fault sensitivity condition}

Based on $c_{r}(k)$ in (19a), the propagation of $c_{f}(k)$ is defined in (18). From Definition 2.4, the $\mathcal{H}_{-}$performance index $\beta$ between the signals $c_{r}(k)$ and $f(k)$ satisfies

$$
\sum_{k=0}^{\infty} c_{r}(k)^{\top} c_{r}(k) \geq \beta^{2} \sum_{k=0}^{\infty} f(k)^{\top} f(k)
$$

Consider that fault occurs in a frequency range $\theta \in$ $\left[\theta_{1}, \theta_{2}\right]$ and the following condition holds

$$
\begin{aligned}
& e^{j \theta_{w}} \sum_{k=0}^{\infty}\left(c_{f}(k+1)-e^{j \theta_{1}} c_{f}(k)\right. \\
& \times\left(c_{f}(k+1)-e^{j \theta_{2}} c_{f}(k)\right)^{\top} \leq 0,
\end{aligned}
$$

with $\theta_{w}=\frac{\theta_{2}-\theta_{1}}{2}$. Based on the generalised KYP lemma (see Lemma 2.5), a relaxation of (28) is given in the following lemma.

Lemma 4.4: Consider the fault frequency contents $\theta \in$ $\Theta$ with $\Theta$ defined in Table 1 , the dynamics of $c_{f}(k)$ in (18) and suppose (TA - GC) to be Schur stable by satisfying (22b). If there exist matrices $P \in \mathbb{S}^{n}$ and $Q \in$ $\mathbb{S}_{\succ 0}^{n}$, and a scalar $\beta>0$ such that

$$
\left[\begin{array}{cc}
T A-G C & T F \\
I_{n} & 0
\end{array}\right]^{\top} \Xi\left[\begin{array}{cc}
T A-G C & T F \\
I_{n} & 0
\end{array}\right]
$$

$$
+\left[\begin{array}{cc}
C & 0 \\
0 & I_{m_{f}}
\end{array}\right]^{\top}\left[\begin{array}{cc}
-I_{p} & 0 \\
0 & \beta^{2} I_{m_{f}}
\end{array}\right]\left[\begin{array}{cc}
C & 0 \\
0 & I_{m_{f}}
\end{array}\right] \prec 0,
$$

where $\Xi$ is chosen as in Table 1 with respect to $\theta \in$ $\Theta$, then the $\mathcal{H}_{-}$performance index $\beta$ between the signals $c_{r}(k)$ and $f(k)$ satisfying $(28)$ holds.

Proof: Without loss of generality, let us first consider $\forall \theta \in \Theta$ in the middle-frequency domain. Recall $\theta_{c}=$ $\frac{\theta_{1}+\theta_{2}}{2}$ and from Table $1, \Pi$ is chosen as

$$
\Pi=\left[\begin{array}{cc}
-P & \star \\
e^{-j \theta_{c}} Q & P-2 \cos \left(\theta_{w}\right) Q
\end{array}\right] .
$$

With (18), pre-multiplying both sides of (30) by $\left[c_{f}(k)^{\top}, f(k)^{\top}\right]$ and post-multiplying it by $\left[c_{f}(k), f(k)\right]$, we obtain a sufficient condition

$$
\begin{gathered}
c_{f}(k)^{\top} P c_{f}(k)-c_{f}(k+1)^{\top} P c_{f}(k+1) \\
-c_{r}(k)^{\top} c_{r}(k)+\beta^{2} f(k)^{\top} f(k) \\
+\operatorname{He}\left(c_{f}(k+1)^{\top} e^{j \theta_{c}} Q c_{f}(k)\right) \\
-c_{f}(k)^{\top} 2 \cos \left(\theta_{w}\right) Q c_{f}(k) \leq 0 .
\end{gathered}
$$

Since the term $\operatorname{He}\left(c_{f}(k+1)^{\top} e^{j \theta_{c}} Q c_{f}(k)\right)-c_{f}(k)^{\top}$ $2 \cos \left(\theta_{w}\right) Q c_{f}(k)$ is a scalar, we have

$$
\begin{aligned}
& \operatorname{He}\left(c_{f}(k+1)^{\top} e^{j \theta_{c}} Q c_{f}(k)\right)-c_{f}(k)^{\top} 2 \cos \left(\theta_{w}\right) Q c_{f}(k) \\
& \quad=\operatorname{tr}\left(Q \left(\operatorname{He}\left(e^{j \theta_{c}} c_{f}(k) c_{f}(k+1)^{\top}\right)\right.\right. \\
& \left.\left.-2 \cos \left(\theta_{w}\right) c_{f}(k) c_{f}(k)^{\top}\right)\right) .
\end{aligned}
$$

Set

$$
\begin{aligned}
S= & \sum_{k=0}^{\infty}\left(\operatorname{He}\left(e^{j \theta_{c}} c_{f}(k) c_{f}(k+1)^{\top}\right)\right. \\
& \left.-2 \cos \left(\theta_{w}\right) c_{f}(k) c_{f}(k)^{\top}\right) .
\end{aligned}
$$

Assuming that the fault appears for a finite period of time in $[0, \infty)$ (i.e. $c_{f}(0)=0$ and $c_{f}(\infty)=0$ ) and by summing (31) from $k=0$ to $\infty$, we obtain

$$
-\sum_{k=0}^{\infty} c_{r}(k)^{\top} c_{r}(k)+\beta^{2} \sum_{k=0}^{\infty} f(k)^{\top} f(k)+\operatorname{tr}(Q S) \leq 0 .
$$

Based on the result of (Iwasaki et al., 2005, Theorem 4), if (29) holds, then we have $\operatorname{tr}(Q S) \geq 0$ and $-\sum_{k=0}^{\infty}$ $c_{r}(k)^{\top} c_{r}(k)+\beta^{2} \sum_{k=0}^{\infty} f(k)^{\top} f(k) \leq 0$ that implies $(28)$. 
Moreover, following the similar procedure of the above proof, by choosing $\theta_{1}=-\theta_{l}$ and $\theta_{2}=\theta_{l}$ for the low-frequency case or $\theta_{1}=-\theta_{h}$ and $\theta_{2}=2 \pi-\theta_{h}$ for the high-frequency case, (28) also holds.

Based on Lemma 4.4, we now build the $\mathcal{H}_{-}$fault sensitivity condition in the following theorem.

Theorem 4.5 ( $\mathcal{H}_{-}$fault sensitivity condition): Given $\left\langle c_{r}(k), H_{r}(k)\right\rangle$ in (13) with $f(k)$ in a finite-frequency domain $\theta_{1} \leq \theta \leq \theta_{2}, \forall k \in \mathbb{N}$, and matrices $T \in \mathbb{R}^{n \times n}$ and $N \in \mathbb{R}^{n \times p}$ satisfying (10). If there exist matrices $W \in \mathbb{S}_{\succ 0}^{n}, Y \in \mathbb{R}^{n \times p}, P \in \mathbb{S}^{n}$ and $Q \in \mathbb{S}_{\succ 0}^{n}$, two scalars $\alpha$ and $\beta>0, L \in \mathbb{R}^{m_{f} \times n}$ such that

$$
\begin{aligned}
& {\left[\begin{array}{c}
\Phi_{f} \\
\alpha F^{\top} T^{\top} W^{\top}+L W T A-L Y C \\
\alpha W^{\top}+e^{j \theta_{c}} Q+W T A-Y C
\end{array}\right.} \\
& \left.\begin{array}{cc}
\star & \star \\
\mathbf{H e}(L W T F)-\beta^{2} I_{m_{f}} & \star \\
W^{\top} L^{\top}+W T F & P+W+W^{\top}
\end{array}\right] \succ 0,
\end{aligned}
$$

with $\Phi_{f}=C^{\top} C-P+2 \cos \left(\theta_{w}\right) Q+\mathbf{H e}(\alpha W T A$ $-\alpha Y C)$, then the $\mathcal{H}_{-}$performance in (28) for the zonotopic FD observer (13) holds.

Proof: For $\theta_{1} \leq \theta \leq \theta_{2}$ in any finite-frequency domain, from (30), we derive

$$
\left[\begin{array}{ll}
\Phi_{1} & \Phi_{2} \\
\Phi_{3} & \Phi_{4}
\end{array}\right] \prec 0
$$

where

$$
\begin{aligned}
\Phi_{1}= & P-2 \cos \left(\theta_{w}\right) Q-C^{\top} C-(T A-G C)^{\top} \\
& \times P(T A-G C) \\
& +\operatorname{He}\left((T A-G C)^{\top} e^{j \theta} Q\right), \\
\Phi_{2}= & e^{-j \theta} Q T F-(T A-G C)^{\top} P T F, \\
\Phi_{3}= & (T F)^{\top} e^{j \theta} Q-(T F)^{\top} P(T A-G C), \\
\Phi_{4}= & \beta^{2} I_{m_{f}}-(T F)^{\top} P T F .
\end{aligned}
$$

Set $\bar{\Phi}=\left[\begin{array}{cc}C^{\top} C-P+2 \cos \left(\theta_{w}\right) Q & 0 \\ 0 & -\beta^{2} I_{m_{f}}\end{array}\right], \quad Q_{f}=\left[\begin{array}{c}Q \\ 0\end{array}\right]$ and $A_{f}=[T A-G C, T F]$. Then, (33) is equivalent to

$$
\left[\begin{array}{c}
I_{n} \\
A_{f}
\end{array}\right]^{\top}\left[\begin{array}{cc}
\bar{\Phi} & e^{-j \theta_{c}} Q_{f} \\
e^{j \theta_{c}} Q_{f}^{\top} & P
\end{array}\right]\left[\begin{array}{c}
I_{n} \\
A_{f}
\end{array}\right] \succ 0 .
$$

By using the Finsler's lemma to above inequality, we obtain

$$
\left[\begin{array}{cc}
\bar{\Phi} & e^{-j \theta_{c}} Q_{f} \\
e^{j \theta_{c}} Q_{f}^{\top} & P
\end{array}\right]+\mathbf{H e}(\bar{R} U) \succ 0,
$$

where $\bar{R} \in \mathbb{R}^{\left(2 n+m_{f}\right) \times n}$ is an arbitrary matrix (called multiplier), and $U=\left[A_{f}, I_{n}\right]$. Then, with $\alpha$ and $L \in$ $\mathbb{R}^{m_{f} \times n}$, we define a structure of the multiplier as

$$
\bar{R}=\left[\begin{array}{c}
\alpha W \\
L W \\
W
\end{array}\right] .
$$

By substituting $\bar{R}$ in (34), we thus obtain (32).

Remark 4.4: From (34), the multiplier $\bar{R}$ can be chosen arbitrarily. Based on the proof of Theorem 4.5, the structure of $\bar{R}$ is defined with the parameters $\alpha$ and $L$ so that the condition (32) is obtained. From the condition (32), the selection of $L$ should satisfy that the term $\mathrm{He}(L W T F)-\beta^{2} I_{m_{f}}$ in the diagonal be strictly positive-definite.

\subsubsection{The optimisation problem setup}

The objective of designing the FD observer gain $G$ is to minimise the effect of uncertainties and maximise the sensitivity to occurred faults. On the one hand, for given $\gamma \in(0,1)$ and $\epsilon>0$, we have the ultimate bound of the $W$-radius $\ell_{W \infty} \leq \frac{\epsilon}{1-\gamma}$ that corresponds to an ellipsoidal set. To minimise the size of this ellipsoid, we can maximise a measure of the matrix $W$, for instance maximising $\operatorname{tr}(W)$. On the other hand, we can maximise the $H_{-}$fault sensitivity index $\beta$.

In general, given $\gamma \in(0,1), \epsilon>0, \alpha, L \in \mathbb{R}^{m_{f} \times n}$, and $T \in \mathbb{R}^{n \times n}$ and $N \in \mathbb{R}^{n \times p}$ satisfying (10), and two prioritisation weights $\lambda_{r}$ and $\lambda_{f}$, the optimisation problem for designing the FD observer gain $G$ can be expressed as follows:

$$
\underset{\substack{W, Y, \Gamma, Y, \Omega, P, Q, \beta^{2}}}{\operatorname{maximize}} \lambda_{r} \operatorname{tr}(W)+\lambda_{f} \beta^{2}
$$

subject to (22a), (22b) and (32).

Then, the optimal solution of (35) provides the optimal FD observer gain by

$$
G=W^{-1} Y \text {. }
$$

Remark 4.5: The weights $\lambda_{r}$ and $\lambda_{f}$ are set for obtaining a trade-off between robustness and fault sensitivity conditions. For instance, the fault sensitivity objective can be enhanced by choosing $\lambda_{f} \geq \lambda_{r}$. 


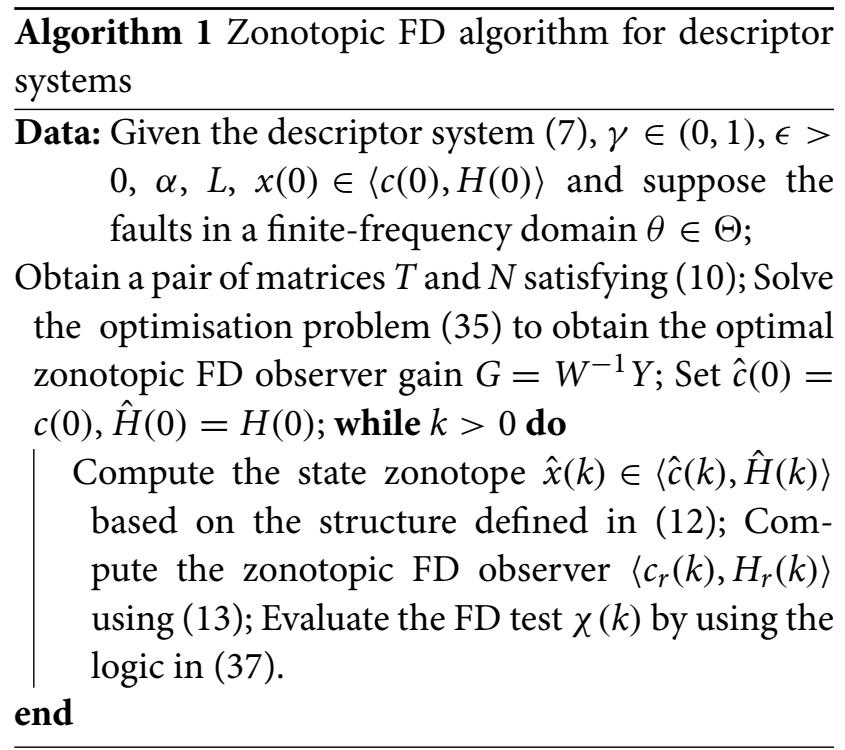

\subsection{Zonotopic fault detection algorithm}

From the output equation (7b), we know $0=y(k)-$ $C x(k)-D_{v} v(k)$. Hence, if $f(k)=0$, we know $0 \in$ $\left\langle c_{r}(k), H_{r}(k)\right\rangle$, which can be used to determine the FD alarm $\chi$. The logic of the FD test $\chi(k), \forall k \in \mathbb{N}$ is formulated as follows:

$$
\chi(k)= \begin{cases}0 & \text { if } 0 \in\left\langle c_{r}(k), H_{r}(k)\right\rangle \\ 1 & \text { if } 0 \notin\left\langle c_{r}(k), H_{r}(k)\right\rangle\end{cases}
$$

where $\chi(k)=0$ means that no fault is detected while $\chi(k)=1$ corresponds to the case that a fault is detected at time step $k$. In general, the robust FD strategy is summarised in Algorithm 1.

\section{Case study: a chemical mixing system}

In this section, we apply the proposed zonotopic FD method to a case study of the chemical mixing system based on the one proposed in Yeu et al. (2005). Comparison with another method for designing the observer gain is also provided to assess the performance of the proposed design method.

\subsection{Description}

By applying the Euler discretization method with the sampling time $T_{s}=0.1 \mathrm{~s}$, we obtain the discrete-time descriptor model of the chemical mixing system as in the form of (7) with system matrices as follows:

$$
\begin{aligned}
& E=\left[\begin{array}{llll}
1 & 0 & 0 & 0 \\
0 & 0 & 0 & 0 \\
0 & 0 & 1 & 0 \\
0 & 0 & 0 & 0
\end{array}\right] \\
& A=\left[\begin{array}{cccc}
0.9625 & 0.0067 & 0 & 0 \\
0 & -0.1 & 0 & 0 \\
0.03 & 0.0533 & 0.95 & -0.004 \\
0 & 0.1 & 0 & -0.1
\end{array}\right] \text {, } \\
& B=\left[\begin{array}{cc}
0.01 & 0 \\
0.1 & 0 \\
0 & 0.002 \\
0 & 0.1
\end{array}\right] \text {, } \\
& B_{w}=\left[\begin{array}{cccc}
0.01 & 0 & 0 & 0 \\
0 & 0.01 & 0 & 0 \\
0 & 0 & 0.01 & 0 \\
0 & 0 & 0 & 0.01
\end{array}\right] \text {, } \\
& C=\left[\begin{array}{llll}
0 & 1 & 0 & 0 \\
0 & 0 & 1 & 0 \\
0 & 0 & 0 & 1
\end{array}\right] \text {, } \\
& D_{v}=\left[\begin{array}{ccc}
0.01 & 0 & 0 \\
0 & 0.01 & 0 \\
0 & 0 & 0.01
\end{array}\right] \text {, }
\end{aligned}
$$

and $F=3 B$. The initial state $x_{0}=[0.5,0,0.5,0]^{\top}$ is assumed to be bounded by the zonotope $x_{0} \in\left\langle c_{0}, H_{0}\right\rangle$, where $c_{0}=x_{0}$ and $H_{0}=0.01 I_{4}$. The input signal $u(k)$ is set as $u(k)=[2,2]^{\top}, \forall k \in \mathbb{N}$. For the reduction operator $\downarrow_{q, W}(\cdot)$, we set $q=20$ and $W$ from the optimal solution of (35). The finite-frequency domain for two faults is considered as $|\theta| \leq 0.1$. From the general solution (11) with $\bar{S}=\mathbf{1}_{4 \times 7}$, we obtain one solution satisfying (10) as follows:

$$
T=\left[\begin{array}{cccc}
1 & 1 & 0 & 1 \\
0 & 1 & 0 & 1 \\
0 & 1 & 0.5 & 1 \\
0 & 1 & 0 & 1
\end{array}\right], \quad N=\left[\begin{array}{ccc}
0 & 0 & 0 \\
1 & 0 & 0 \\
0 & 0.5 & 0 \\
0 & 0 & 1
\end{array}\right]
$$

\subsection{Simulation results}

First, we discuss the trade-off achieved with different selections of weights $\lambda_{r}$ and $\lambda_{f}$. The objective of the optimisation problem (35) can be normalised considering only a goal a time by selecting $\lambda_{r}=0$ and $\lambda_{f}=0$, respectively. The optimal solutions for these two cases are reported at the beginning and the end of 
Table 2. Comparison of robustness and fault sensitivity performances with different weights.

\begin{tabular}{lcccc}
\hline Weights & $\begin{array}{c}\text { Robustness } \\
\operatorname{tr}(W)\end{array}$ & $\begin{array}{c}\text { Fault sensitivity } \\
\beta\end{array}$ & MDF $a_{1}$ & MDF $a_{2}$ \\
\hline$\lambda_{r}=0.25, \lambda_{f}=0.75$ & 771.85 & 0.1249 & 0.34 & 0.35 \\
$\lambda_{r}=0.5, \lambda_{f}=0.5$ & 775.79 & 0.1179 & 0.28 & 0.27 \\
$\lambda_{r}=0.75, \lambda_{f}=0.25$ & 778.99 & 0.0927 & 0.2 & 0.23 \\
$\lambda_{r}=1, \lambda_{f}=0$ & 779.83 & - & 0.22 & 0.2 \\
\hline
\end{tabular}

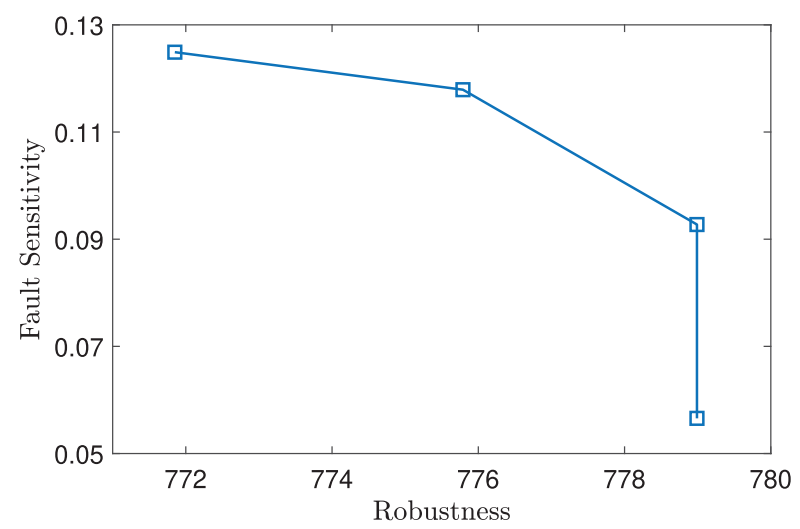

Figure 1. Comparison of robustness and fault sensitivity with different weights.

Table 2. After the normalisation, both weights $\left(\lambda_{r}, \lambda_{f}\right)$ can be chosen $\in[0,1]$. We have carried out the simulations with different values of these weights in this range and the comparisons are shown in Table 2 to show the trade-off between robustness against uncertainties and sensitivity to faults. From the results of robustness and fault sensitivity in Table 2, the robustness index is increasing as the weight $\lambda_{r}$ increases while the fault sensitivity index is decreasing as the weight $\lambda_{f}$ decreases. As shown in Figure 1, it is obvious that a good trade-off between two performance indexes is required.

To assess the performance of the proposed approach, let consider faults in the two actuators $f_{1}(k)=$

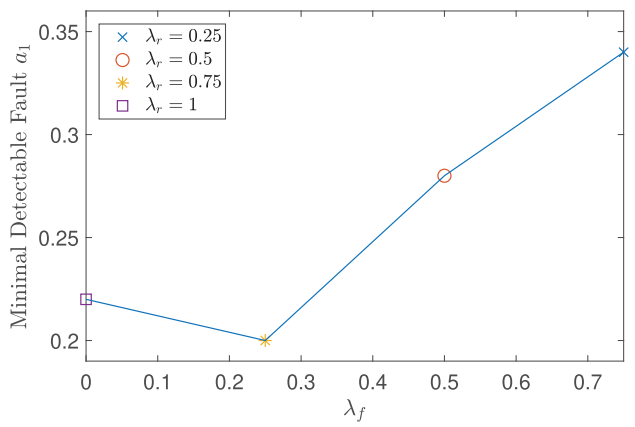

(a) $\left.\left[a_{1} \sin (\theta k), 0\right)\right]^{\top}$ and $f_{2}(k)=\left[0, a_{2} \sin \left(\theta_{2} k\right)\right]^{\top}$ appearing in a sequential manner and lasting each fault for 30 time steps, where $\theta=0.05 \leq 0.1$. By means of simulations, we can evaluate the minimal detectable fault (MDF) magnitude, i.e. the minimum value of $a_{1}$ or $a_{2}$ that allow detecting the faults for at least one-step. The results of minimal detectable faults are also presented in Table 2. As shown in Figure 2, the minimal detectable faults $a_{1}$ and $a_{2}$ are plotted with different selections of weights $\lambda_{r}$ and $\lambda_{f}$. From this comparison, it is clear that a trade-off between two objectives is very important and has impact on the MDF. As labeled in the yellow stars in both plots of Figure 2, we can realize that the selection of weights $\lambda_{r}=0.75$ and $\lambda_{f}=0.25$ is a good trade-off since MDF $a_{1}$ is the smallest and MDF $a_{2}$ is relatively small compared to the other selections.

We next present the simulation results with different faulty cases. Several time-varying faults with different frequencies (not occurred simultaneously) are considered as follows:

$$
\begin{array}{ll}
f_{1}(k) & =\left[a_{1} \sin \left(\theta_{1} k\right), 0\right]^{\top} \quad k_{1} \leq k \leq k_{2}, \\
f_{2}(k) & =\left[0, a_{2} \sin \left(\theta_{2} k\right)\right]^{\top} \quad k_{3} \leq k \leq k_{4},
\end{array}
$$

where $\theta_{1}$ and $\theta_{2}$ are the frequencies for two faults, respectively.

Based on the development of Theorem 4.3, we choose $\gamma=0.5, \epsilon=1$ to guarantee the convergence of the size of zonotopes. According to Remark 4.5 and the results presented in Table 2, we choose $\lambda_{r}=0.75$ and $\lambda_{f}=0.25$ to make the zonotopic FD observer more sensitive. With these parameters, $\alpha=0.3$ and $L=30 F^{\top}$ are adjusted from the feasibility of the optimization problem (35). In the simulation results presented in the following, we consider two faulty cases as follows:

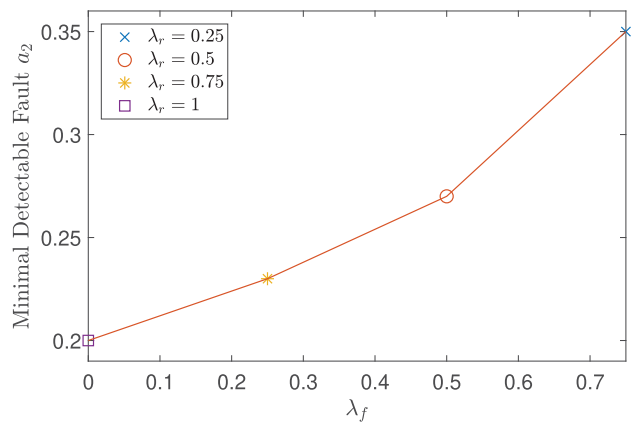

(b)

Figure 2. Comparison of minimal detectable faults with different weights. (a) MDF $a_{1}$. (b) MDF $a_{2}$. 


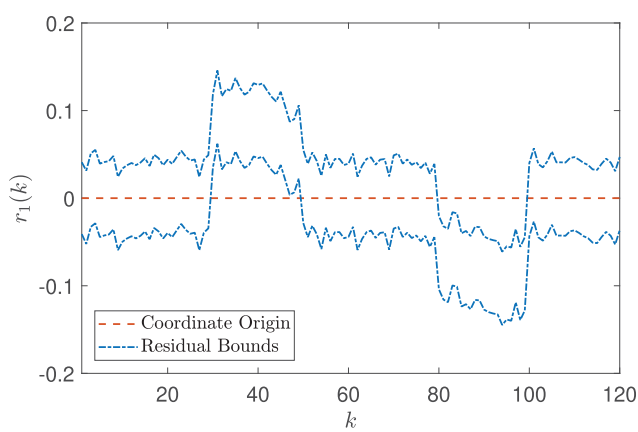

(a) $r_{1}$

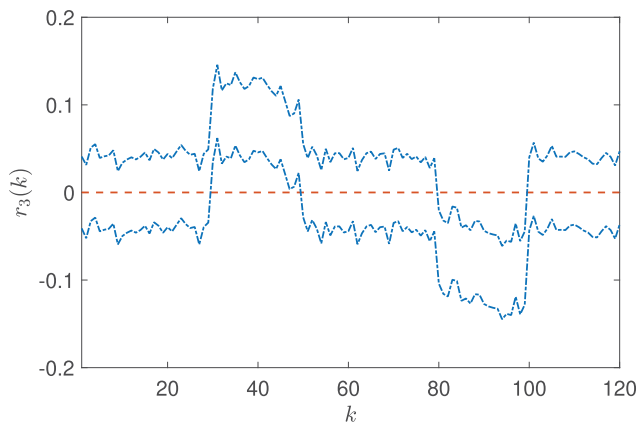

(c) $r_{3}$

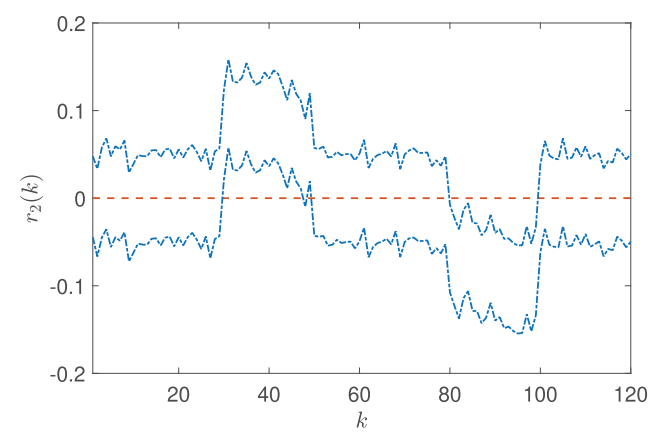

(b) $r_{2}$

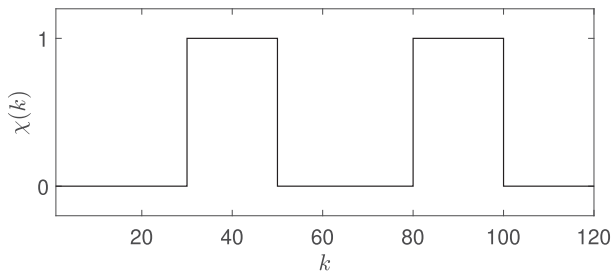

(d) $\chi$

Figure 3. Case 1: fault detection result with $\theta_{1}=\theta_{2}=0.05$ and $a_{1}=a_{2}=0.25$. (a) $r_{1}$. (b) $r_{2}$. (c) $r_{3}$. (d) $\chi$.

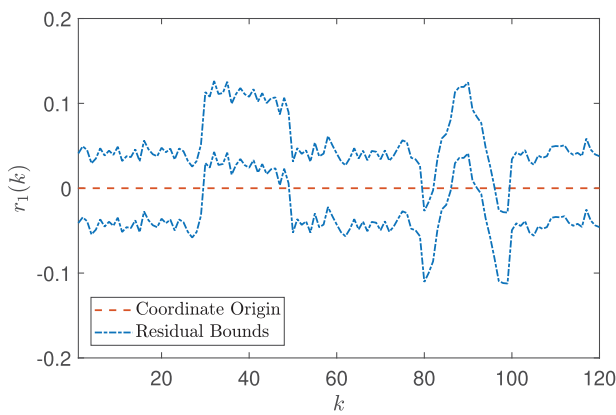

(a) $r_{1}$

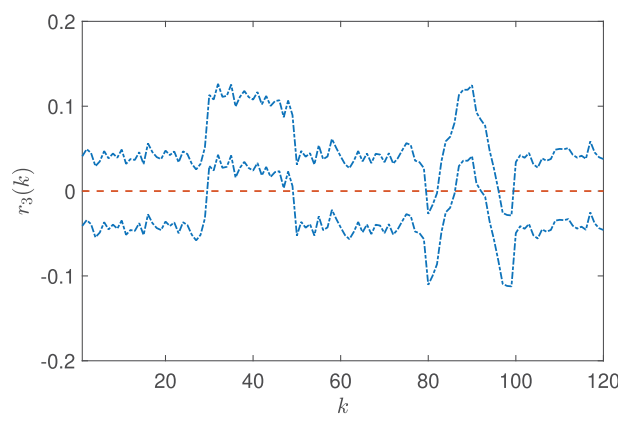

(c) $r_{3}$

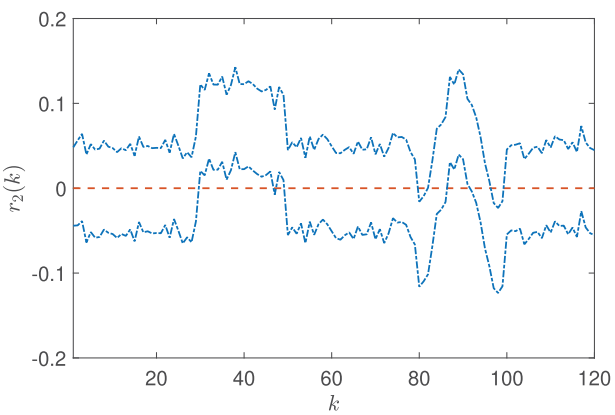

(b) $r_{2}$

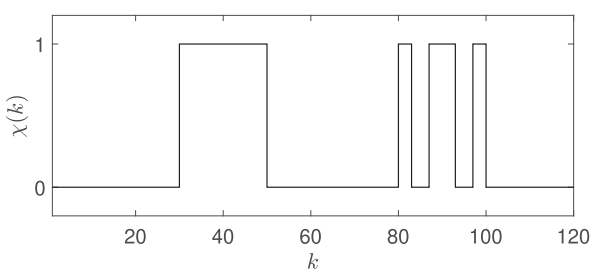

(d) $\chi$

Figure 4. Case 2: fault detection result with $\theta_{1}=0.05, \theta_{2}=0.3$ and $a_{1}=a_{2}=0.25$. (a) $r_{1}$. (b) $r_{2}$. (c) $r_{3}$. (d) $\chi$. 
- Case 1: $\left|\theta_{1}\right|=\left|\theta_{2}\right| \leq 0.1$

- Case 2: $\left|\theta_{1}\right| \leq 0.1,\left|\theta_{2}\right| \geq 0.1$ and $a_{1}=a_{2}$.

For each case, the residual bound $r_{i} \in\left[\underline{r}_{i}, \bar{r}_{i}\right]$ for $i=$ $1, \ldots, p$ and $\forall k \in \mathbb{N}$ is computed by using the interval hull of the zonotope (as in Definition 2.2) determined by

$$
\begin{aligned}
& \underline{r_{i}}=c_{r, i}-r s\left(H_{r}\right)_{i, i}, \\
& \overline{r_{i}}=c_{r, i}+r s\left(H_{r}\right)_{i, i} .
\end{aligned}
$$

Fault detection results are shown in Figure 3-4. In Case 1 , the frequencies $\theta_{1}$ and $\theta_{2}$ for both faults are within the range $|\theta| \leq 0.1$ where the observer achieves the best trade-off between fault sensitivity and robustness. As shown in Figure 3, the faults occurred during two periods $30 \leq k \leq 50$ and $80 \leq k \leq 100$ are detected with $a_{1}=a_{2}=0.25$. In Case 2, $\theta_{1}=0.05$ still remains in the given frequency domain and $\theta_{2}$ is considered to be out of this given domain. In Figure 4, with $\theta_{2}=0.3$, the residual signals are moving around the coordinate origin so that some faults occurred between $83 \leq k \leq$ 86 and $93 \leq k \leq 96$ cannot be detected.

\subsection{Comparison with other method}

The performance of the proposed FD observer gain is compared with a time-varying observer gain denoted by $\bar{G}(k)$ obtained only to optimise state estimation performance. Following the method in Wang et al. (2018, Theorem 6), this time-varying observer gain can be obtained by

$$
\bar{G}(k)=T A \bar{P}(k) C^{\top}\left(C \bar{P}(k) C^{\top}+D_{v} D_{v}^{\top}\right)^{-1},
$$

with $\bar{P}(k)=\hat{H}(k) \hat{H}(k)^{\top}$.

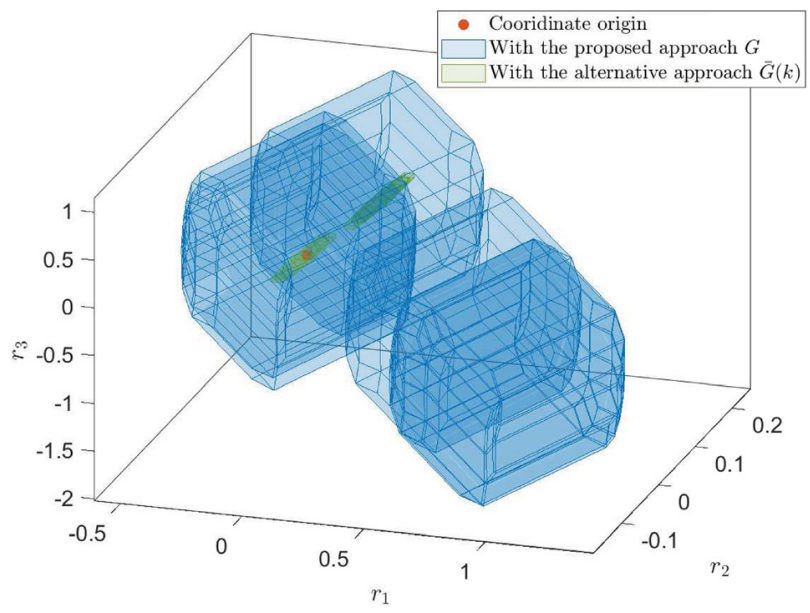

Figure 5. Comparison of the residual zonotopes.
In this case, the objective is only to minimise the effect of uncertainties corresponding to the state bounding zonotope $\langle\hat{c}(k), \hat{H}(k)\rangle, \forall k \in \mathbb{N}$. The residual zonotopes (13) from time step $k=48$ to $k=53$ are shown in Figure 5. From these zonotopes, it can be seen that the size of these zonotopes does not diverge. Besides, the residual zonotopes obtained with the optimal FD observer gain are more sensitive to faults since these zonotopes are located away from the coordinate origin.

\section{Conclusion}

In this paper, we have designed a zonotopic FD observer for discrete-time descriptor systems affected by actuator faults. Under a set-based framework of descriptor systems, we have decomposed the state bounding zonotope in two zonotopes to separate the effects of uncertainties and faults. Taking into account the FD objective, fault sensitivity has been assessed based on the decomposed zonotopes and a $\mathcal{H}_{-}$index. To design the optimal FD observer gain, we have obtained two LMI conditions that allow establishing the trade-off robustness against uncertainties and sensitivity to faults, respectively. Then, based on these conditions, the optimal FD observer gain can be obtained by solving an offline optimisation problem. Finally, we have applied the proposed method to a case study to show its effectiveness. The comparison results with an approach that does not consider the trade-off between robustness and fault sensitivity have been provided. Besides, it is worth mentioning that with $\operatorname{rank}(E)=n$, the proposed setbased FD method can also be used for discrete-time dynamic systems. As future work, we will explore relaxing the observability conditions of the proposed method.

\section{Acknowledgements}

This work was partially funded by the Fundamental Research Funds for the Central University under the project (No. 3072020CFJ0401), and by the Natural Science Foundation of Heilongjiang Province under the grant (YQ2020F008).

\section{Disclosure statement}

No potential conflict of interest was reported by the authors. 


\section{Funding}

This work was partially funded by the Fundamental Research Funds for the Central University under the project [grant number 3072020CFJ0401], and by the Natural Science Foundation of Heilongjiang Province under the grant [grant number YQ2020F008].

\section{Notes on contributors}

Ye Wang received the M.Sc. degree in Automatic Control and Robotics and the Ph.D. degree (Cum Laude) in Automatic Control, Robotics, and Vision both from the Universitat PoliteİĂcnica de Catalunya-BarcelonaTech (UPC), Spain, in 2014 and 2018, respectively. From 2015 to 2018, he was a Research Fellow of the Spanish National Research Council (CSIC) at the Institut de RoboİĂtica i InformaİĂtica Industrial (IRI), CSIC-UPC. In March 2016, November 2016 and January 2018, he was a visiting researcher at University of Seville, Spain. From February to May 2017, and from March to April 2018, he was a visiting researcher at CentraleSupélec, University of Paris-Saclay, France. $\mathrm{He}$ is an Associate Professor at the College of Intelligent Systems Science and Engineering, Harbin Engineering University (HEU), China. His research interest includes model predictive control, fault diagnosis and fault-tolerant control.

VicencĬğ Puig received the B.Sc. and M.Sc. degrees in Telecommunications Engineering and the Ph.D. degree in Automatic Control, Vision, and Robotics from the Universitat PoliteİĂcnica de Catalunya-BarcelonaTech (UPC), Barcelona, Spain, in 1993 and 1999, respectively. He is a Full Professor at the Automatic Control Department, UPC, and a researcher at the Institut de RoboİĂtica i InformaİĂtica Industrial (IRI), CSIC-UPC. $\mathrm{He}$ is also the director of the Automatic Control Department and the head of the Advanced Control Systems (SAC) research group, UPC. He has developed important scientific contributions in the areas of fault diagnosis and fault-tolerant control, using interval, and linear-parameter-varying models using setbased approaches. He has participated in more than 20 European and national research projects in the last decade. He has also led many private contracts with several companies and has published more than 220 journal articles as well as over 500 contributions in international conference/workshop proceedings. He has supervised over 25 Ph.D. dissertations and over 50 master theses/final projects. He was the general chair of the 3rd IEEE Conference on Control and Fault-Tolerant Systems (SysTol 2016) and the IPC chair of IFAC Safeprocess 2018. He is also the chair of the IFAC Safeprocess TC Committee 6.4.

Gabriela Cembrano received the M.Sc. degree in Industrial Engineering and the Ph.D. degree in Automatic Control from the Universitat Politècnica de Catalunya-BarcelonaTech (UPC). She is a tenured researcher of the Spanish National Research Council (CSIC) at the Institut de Robòtica i Informàtica Industrial (IRI), CSIC-UPC. Since 2007, she has been a scientific advisor of the CETaqua, a water technology centre. Her main research interest includes control engineering and its applications to water systems management. She has taken part in numerous fundamental and industrial research projects in this field since 1990. Most recently, she has been the scientific director of the EC Project EFFINET and the CSIC leader in projects LIFE-EFFIDRAIN and DEOCS. She has published over 150 journal and conference papers in this field.

Yuxin Zhao received the Ph.D. degree in Navigation, Guidance and Control from the Harbin Engineering University (HEU) in 2005, and completed a postdoctoral research in Control Science and Engineering at the Harbin Institute of Technology (HIT) in 2008. In 2004, he was awarded a scholarship for visiting the Levin Institute of State University of New York (SUNY) from the State Administration of Foreign Experts Affairs. From 2012 to 2013, he was a visiting scholar at the Centre for Transport Studies (CTS), Imperial College London. He is a Full Professor and Dean of the College of Intelligent Systems Science and Engineering, HEU, China. His research interest includes artificial intelligence, filtering theory, marine navigation system and intelligent transportation system. He has published more than 100 papers, including more than 40 international journal papers in these areas.

\section{References}

Alamo, T., Bravo, J., \& Camacho, E. (2005). Guaranteed state estimation by zonotopes. Automatica, 41(6), 1035-1043. https://doi.org/10.1016/j.automatica.2004.12.008

Biegler, L., Campbell, S., \& Mehrmann, V. (2012). Control and optimisation with differential-algebraic constraints. Society for Industrial and Applied Mathematics.

Blanke, M., Kinnaert, M., Lunze, J., \& Staroswiecki, M. (2016). Diagnosis and fault-tolerant control. Springer.

Chabane, S., Stoica, C., Alamo, T., Camacho, E. F., \& Dumur, D. (2014). Improved set-membership estimation approach based on zonotopes and ellipsoids. In 2014 European control conference (ECC), Strasbourg, France (pp. 993-998). IEEE.

Chadli, M., Abdo, A., \& Ding, S. (2013). Fault detection filter design for discrete-time Takagi-Sugeno fuzzy system. Automatica, 49(7), 1996-2005. https://doi.org/10.1016/ j.automatica.2013.03.014

Chen, J., \& Patton, R. (1999). Robust model-based fault diagnosis for dynamic systems. Springer.

Combastel, C. (2015). Zonotopes and Kalman observers: Gain optimality under distinct uncertainty paradigms and robust convergence. Automatica, 55, 265-273. https://doi.org/ 10.1016/j.automatica.2015.03.008

Ding, S. (2013). Model-based fault diagnosis techniques. Springer. Duan, G. (2010). Analysis and design of descriptor linear systems. Springer.

Goupil, P. (2010). Oscillatory failure case detection in the A380 electrical flight control system by analytical redundancy. Control Engineering Practice, 18(9), 1110-1119. https://doi.org/10.1016/j.conengprac.2009.04.003

Iwasaki, T., \& Hara, S. (2005). Generalized KYP lemma: Unified frequency domain inequalities with design applications. IEEE Transactions on Automatic Control, 50(1), 41-59. https://doi.org/10.1109/TAC.2004.840475 
Iwasaki, T., Hara, S., \& Fradkov, A. (2005). Time domain interpretations of frequency domain inequalities on (semi)finite ranges. Systems \& Control Letters, 54(7), 681-691. https:// doi.org/10.1016/j.sysconle.2004.11.007

Komachali, F., \& Shafiee, M. (2020). Sensor fault diagnosis in fractional-order singular systems using unknown input observer. International Journal of Systems Science, 51(1), 116-132. https://doi.org/10.1080/00207721.2019.1701135

Komachali, F., Shafiee, M., \& Darouach, M. (2019). Design of unknown input fractional order proportional-integral observer for fractional order singular systems with application to actuator fault diagnosis. IET Control Theory \& Applications, 13(14), 2163-2172. https://doi.org/10.1049/ietcta.2018.5712

Le, V., Stoica, C., Alamo, T., Camacho, E., Dumur, D. (Eds.). (2013). Zonotopes from guaranteed state-estimation to control. John Wiley \& Sons.

Merhy, D., Stoica, C., Alamo, T., \& Camacho, E. F. (2019). Ellipsoidal set-membership state estimation for descriptor systems. In 2019 23rd International conference on system theory, control and computing (ICSTCC), Sinaia, Romania (pp. 1-6). IEEE.

Nosrati, K., \& Shafiee, M. (2018). Kalman filtering for discretetime linear fractional-order singular systems. IET Control Theory \& Applications, 12(9), 1254-1266. https://doi.org/ 10.1049/iet-cta.2017.0898

Puig, V. (2010). Fault diagnosis and fault tolerant control using set-membership approaches: application to real case studies. International Journal of Applied Mathematics and Computer Science, 20(4), 619-635. https://doi.org/10.2478/v10006-010 -0046-y

Puig, V., Quevedo, J., Escobet, T., \& Nejjari, F. (2008). Passive robust fault detection of dynamic processes using interval models. IEEE Transactions on Control Systems Technol- ogy, 16(5), 1083-1089. https://doi.org/10.1109/TCST.2007. 906339

Stevens, B., Lewis, F., \& Johnson, E. (2016). Aircraft control and simulation: Dynamics, controls design, and autonomous systems. Wiley-Blackwell.

Varga, A. (2017). Solving fault diagnosis problems. Springer.

Wang, Y., Puig, V., \& Cembrano, G. (2017). Non-linear economic model predictive control of water distribution networks. Journal of Process Control, 56, 23-34. https:// doi.org/10.1016/j.jprocont.2017.05.004

Wang, Y., Puig, V., \& Cembrano, G. (2018). Set-membership approach and Kalman observer based on zonotopes for discrete-time descriptor systems. Automatica, 93, 435-443. https://doi.org/10.1016/j.automatica.2018.03.082

Wang, Z., Shen, Y., Zhang, X., \& Wang, Q. (2012). Observer design for discrete-time descriptor systems: An LMI approach. Systems \& Control Letters, 61(6), 683-687. https://doi.org/ 10.1016/j.sysconle.2012.03.006

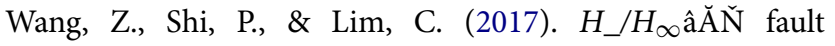
detection observer in finite frequency domain for linear parameter-varying descriptor systems. Automatica, 86, 38-45. https://doi.org/10.1016/j.automatica.2017.08.021.

Wang, Y., Zhou, M., Puig, V., Cembrano, G., \& Wang, Z. (2017). Zonotopic fault detection observer with $H_{-}$performance. In Chinese control conference (CCC), Dalian, China (pp. 7230-7235). IEEE.

Xu, F., Puig, V., Ocampo-Martinez, C., Stoican, F., \& Olaru, S. (2014). Actuator-fault detection and isolation based on set-theoretic approaches. Journal of Process Control, 24(6), 947-956. https://doi.org/10.1016/j.jprocont.2014.04.016

Yeu, T., Kim, H., \& Kawaji, S. (2005). Fault detection, isolation and reconstruction for descriptor systems. Asian Journal of Control, 7(4), 356-367. https://doi.org/10.1111/asjc.2005.7. issue- 4 